\title{
Diacronie
}

Studi di Storia Contemporanea

$N^{\circ} 12,4 \mid 2012$

Sulle tracce delle idee

\section{L'esperienza intellettuale e politica del laburismo nel pensiero di Carlo Rosselli}

\section{Michele Mioni}

\section{Q OpenEdition \\ 1 Journals}

\section{Edizione digitale}

URL: http://journals.openedition.org/diacronie/2535

DOI: $10.4000 /$ diacronie. 2535

ISSN: 2038-0925

\section{Editore}

Association culturelle Diacronie

\section{Notizia bibliografica digitale}

Michele Mioni, «L'esperienza intellettuale e politica del laburismo nel pensiero di Carlo Rosselli », Diacronie [Online], N 12, 4 | 2012, documento 8, Messo online il 29 décembre 2012, consultato il 12 juin 2020. URL : http://journals.openedition.org/diacronie/2535 ; DOI : https://doi.org/10.4000/ diacronie. 2535 


\section{Diacronie}

\section{L'esperienza intellettuale e politica del}

laburismo nel pensiero di Carlo Rosselli

Michele MIONI*

Tra le influenze dell'eclettica formazione politica di Carlo Rosselli, una delle più importanti è stata probabilmente quella derivante dall'esperienza intellettuale e politica del fabianesimo e del laburismo inglese. Questa duplice influenza è individuabile nel pensiero politico ed economico di Rosselli e nella sua peculiare visione del partito politico.

Anche dal confronto con il pensiero politico fabiano, Rosselli ha sviluppato le sue considerazioni sulla centralità del sindacalismo nell'organizzazione economicoindustriale, e come momento di "autogestione" operaia.

L'azione politica del partito laburista, invece, sembrava confermare la sua convinzione che le regole democratiche e parlamentari fossero l'unico mezzo di progresso del socialismo nella società, attraverso il partito e il sindacato, i quali raccoglievano la "funzione liberale" affidata, in quel momento storico, al proletariato. Il partito laburista, in questo senso, sarebbe dovuto essere un esempio per i socialisti democratici e i marxisti italiani, bloccati nell'azione politica dal loro dottrinarismo.

\section{Introduzione}

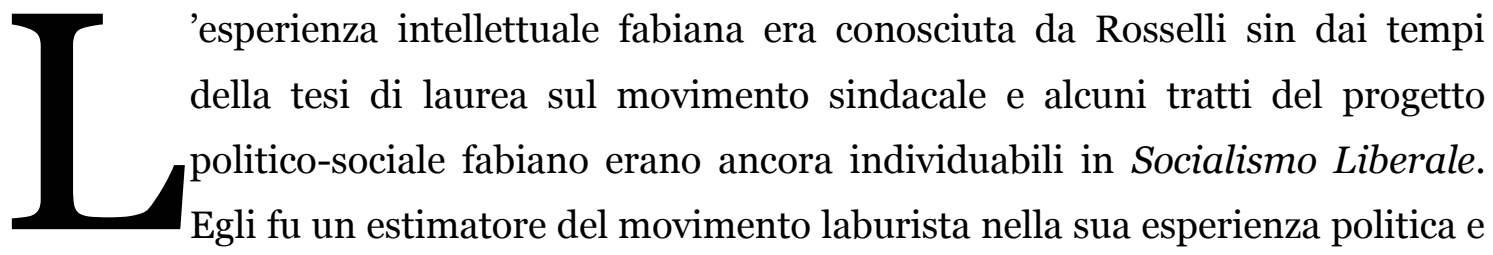
sindacale, per via del suo empirismo non-marxista e per il suo gradualismo politico. 
La sua conoscenza indiretta del movimento operaio britannico sarebbe stata approfondita in due brevi periodi trascorsi in Gran Bretagna per motivi di studio, nel 1923 e nel 1924.

Si cercherà in questa sede di individuare i tratti caratterizzanti del pensiero politico fabiano - sia nella sua accezione politico-ideologica, sia in quella più prettamente economica - che hanno influenzato il pensiero politico di Carlo Rosselli.

L'ammirazione per il laburismo tuttavia non si fermava al solo aspetto programmatico, ma era stata rafforzata dalla politica del partito guidato da MacDonald, che, proprio nel 1924 - quando Rosselli si trovava in Gran Bretagna - conosceva il "salto di qualità" nella politica britannica, ponendosi al tempo stesso come il partito "del Lavoro" e come forza di governo affidabile e matura in una democrazia liberale.

\section{Il Fabianesimo e la "funzione" democratica del socialismo}

Nella formazione intellettuale del giovane Carlo Rosselli, la cultura politica inglese ha giocato un ruolo di fondamentale importanza, sia sul versante radicale e liberalsocialista, sia per quello più marcatamente socialistico e tradunionista ${ }^{1}$. In questo secondo filone politico, è possibile rintracciare un'influenza più o meno diretta del pensiero fabiano, anche per quel che riguarda la teoria sindacale. Rosselli avrebbe conosciuto l'opera dei coniugi Webb e, quasi allo spettro opposto del variegato movimento, le tendenze sindacaliste gildiste di G.D. Howard Cole ${ }^{2}$.

Il fabianesimo affondava le sue radici nel mondo socialista inglese di fine XIX secolo, ancora non costituitosi partito, legato a doppio filo al radicalismo politico e, almeno in parte, estraneo alle contemporanee esperienze europee. In origine, la Fabian Society fu poco più che un cenacolo che riuniva periodicamente professionisti, intellettuali, artisti, operai specializzati, e un numero relativamente elevato di donne3.

\footnotetext{
${ }^{1}$ Per una panoramica sui principali teorici del liberalismo radicale e socialista inglese, si veda URBINATI, Nadia, Il liberalismo socialista nella tradizione inglese, in BOVERO, Michelangelo, MURA, Vincenzo, SBARBERI, Franco (a cura di), I dilemmi del liberalsocialismo, Roma, La Nuova Italia Scientifica, pp. 211-235.

2 Sull'influenza che la cultura politica inglese ha avuto su Carlo Rosselli, sia in termini di elaborazione intellettuale, sia di strategia politica, fino almeno a Socialismo Liberale, si veda anzitutto MASTELLONE, Salvo, Carlo Rosselli e "la rivoluzione liberale del socialismo", Firenze, Olschki, 1999, cfr. in particolare pp. 21-55.

3 Sulla nascita della Fabian Society, si veda l'introduzione all'edizione americana del 1911 dei Fabian Essays di CLARKE, William, La società fabiana, in SHAW, George Bernard, et al., Saggi fabiani, Roma, Editori Riuniti, 1990, pp. 241-260. Sulla genesi del movimento fabiano, e sugli "antecedenti" teorici del movimento nel radicalismo inglese e nel pensiero di John Stuart Mill, si veda PENCH, Lucio Renzo, Il socialismo fabiano: un collettivismo non marxista, Napoli, Edizioni Scientifiche Italiane, 1988. Per una storia del movimento fabiano fino agli anni
} 
Il programma politico fabiano alla fine del XIX secolo era fondamentalmente espresso nei Fabian Essays in Socialism4; questa raccolta di saggi è divisa in due parti: la prima si sofferma sull'analisi delle basi storiche, economiche e morali per cui il socialismo si sarebbe dovuto rivelare "necessario", mentre la seconda disegna l'idea di società socialista che sarebbe venuta.

Le motivazione che avrebbe reso ineluttabile il socialismo si riteneva fosse l'insostenibilità di un sistema che non aumentava la ricchezza della società, bensì le ricchezze dei patrimoni dei singoli capitalisti. Il paradosso di fondo era cioè costituito dall'aumento esponenziale delle capacità produttive e la moltiplicazione proporzionale della miseria delle classi subalterne 5 . Secondo l'analisi di William Clarke era perciò in atto un processo non riconducibile ad una rivoluzione politica sul modello francese, quanto piuttosto ad una lenta trasformazione economico-sociale, «That great change is proceeding silently every day» 6 .

Nel capitalismo di fine XIX secolo erano oramai introdotti alcuni elementi di "socialismo" attraverso la progressiva diminuzione della concorrenza, la concentrazione della produzione in monopoli o "cartelli” industriali e commerciali e l’intervento dello Stato, per esempio in materia di libertà sindacale. Era questo un socialismo "statalistico" e gradualista, che alla fine avrebbe prevalso anche in materia di proprietà dei mezzi di produzione7. Statalismo che peraltro non era sinonimo di centralizzazione, ma piuttosto socializzazione dei servizi fondamentali erogati dai Comuni: il trasporto pubblico locale, le reti idriche e le industrie manifatturiere locali, il sostegno dei disoccupati non attraverso l'assistenzialismo, bensì con il reintegro nelle attività produttive e nel tessuto sociale locale. Allo Stato erano assegnati i compiti di gestione delle grandi reti e dei servizi di comunicazione nazionale, come le ferrovie, le poste e le

Cinquanta si veda COLE, Margaret, The Story of Fabian Socialism, Stanford, Stanford University Press, 1961, cfr. in particolare sui Fabian Essays pp. 26-33. Si veda anche COLE, Howard G. D., Storia del movimento operaio inglese, Vol. II, 190o-1947, Milano, Bonetti, 1965, cfr. pp. 20-30; BEVIR, Mark, The Making of British Socialism, Princeton, Princeton University Press, 2011, cfr. pp. 131-214.

4 SHAW, George Bernard, et al., Fabian Essays in Socialism, London, Walter Scott, 1911.

${ }^{5}$ Per l'analisi economica del capitalismo, si veda SHAW, George Bernard, Economic, in SHAW, George Bernard, et. al., Fabian Essays in Socialism, cit., pp. 3-29.

${ }^{6}$ CLARKE, William, Industrial, in SHAW, George Bernard, et. al., Fabian Essays in Socialism, cit., pp. 62-101, cit. p. 62.

7 È stato notato come tale concezione "positivista" del socialismo presente nei Fabian Essays si basasse sulla convinzione che l'evoluzione dell'"organismo sociale" nell'epoca dell'industrializzazione avesse assunto i caratteri progressivi - ma sempre più evidenti - di un'estensione del ruolo dello Stato dalla sfera "politica" a quella "economico-sociale", con l'assunzione di compiti sempre maggiori di controllo e di gestione della produzione industriale. Tale tendenza, secondo i fabiani - e particolarmente secondo Sidney Webb-, comportava un implicito e graduale abbandono del laissez faire verso nuove forme di organizzazione sociale "comunitarie" e collettiviste. Si veda PENCH, Lucio Renzo, op. cit., cfr. pp. 84-90. 
grandi industrie come quelle minerarie. L'ideale collettivistico fabiano era anche un ideale socialista "municipale"8.

Più importante nel percorso che stiamo qui cercando di tracciare è però il contributo di Sidney Webb, volto a rintracciare le "basi storiche" del socialismo". Nell'ideale di una società dinamica e in evoluzione attraverso il legame storico tra democrazia e socialismo ${ }^{10}$, l'uno era il completamento dell'altro, poiché la democrazia, se ristretta al semplice aspetto politico senza essere riformata socialmente ed economicamente, sarebbe rimasta un semplice meccanismo formale: «advocates of social reconstruction have learnt the lesson of democracy, and know that is through the slow and gradual turning of the popular mind to new principles that social reorganization bit by bit comes» ${ }^{11}$.

La nuova sintesi politica esposta da Webb intendeva integrare il radicalismo democratico con il socialismo economico, di pari passo con la fine dell'individualismo del laissez faire per una nuova organizzazione di stampo marcatamente collettivistico della vita sociale ed economica. Essa avrebbe sostituito al paradigma dell'individuo che gode di libertà assolute e contrapposte agli interessi sociali, come nel caso della "sacralità" della proprietà privata, la consapevolezza dell'uomo all'interno della società, che sarebbe stato tanto più libero quanto più l'organizzazione politica ed economica sarebbe stata democratica e socialista ${ }^{12}$.

Per giungere a questo obiettivo, la strada maestra era quella della socializzazione della proprietà privata e dell'industria, ma anche quella delle riforme sociali, come l'istruzione pubblica e il limite agli orari di lavoro, che costituivano per i fabiani una vera e propria educazione sociale e morale dei lavoratori, alla base di una società anche eticamente socialista $^{13}$. Il fine ultimo di questo cambiamento doveva essere l'instaurazione della socialdemocrazia (o meglio, della piena realizzazione del socialismo inteso come naturale "evoluzione" della democrazia $\left.{ }^{14}\right)$; tuttavia, se il

\footnotetext{
${ }^{8}$ BESANT, Annie, Industry under Socialism, in SHAW, George Bernard, et. al., Fabian Essays in Socialism, cit., pp. 150-169.

${ }^{9}$ WEBB, Sidney, Historic, in SHAW, George Bernard, et. al., Fabian Essays in Socialism, cit., pp. 30-61.

${ }^{10}$ Ibidem, p. 33 e seg.

${ }^{11}$ Ibidem, p. 34.

${ }^{12}$ Ibidem, pp. 56-61.

${ }^{13}$ OLIVIER, Sidney, Moral, in SHAW, George Bernard, et. al., Fabian Essays in Socialism, cit., pp. 102-128, cfr. in particolare pp. 125-128.

${ }_{14}$ Tale concetto ricorrente nei Fabian Essays è sottolineato in particolare da Sidney Webb e George Bernard Shaw. Si vedano WEBB, Sidney, Historic, in cit., in cui l'autore afferma che «[...] as Democracy in political administration continue to be the dominant principle, Socialism may be quite safely predicted as its economic observe, [...]», p. 63; SHAW, George Bernard, Transition, in ID., et. al., Fabian Essays in Socialism, cit., pp. 173-201, il quale si chiede retoricamente «What then does a gradual transition to Social Democracy mean specifically? It
} 
socialismo «is as honest as it is inevitable» era anche vero che «all the mobs and guillotines in the world can no more establish it than police coercion can avert it» ${ }^{15}$.

I fabiani erano convinti che solo nella democrazia potesse essere attuato il socialismo, distinguendo cosi il social-democratico, dal semplice democratico formale, che vedeva nella democrazia il fine ultimo e non il mezzo per la transizione. Le soluzioni "catastrofiste" erano decisamente abbandonate nel fabianesimo a favore di un'idea di transizione che consistesse dapprima nell'estensione della democrazia, per esempio attraverso il diritto di voto, e, in seguito, in una serie di misure economiche che gradualmente, usando gli strumenti democratici della tassazione e delle nazionalizzazioni dei servizi pubblici, spostassero la proprietà dei mezzi di produzione dai privati ad uno Stato che, secondo Bernard Shaw, avrebbe dovuto essere in sostanza centrato sull'autogoverno locale come nucleo di un vero Stato socialdemocratico ${ }^{16}$.

Un altro saggio fabiano ${ }^{17}$ affrontava quella che era la vera questione politica britannica in quegli anni; da un lato il progresso in senso socialista della vita economica del Paese, e dall'altro il conservatorismo del sistema politico inglese incentrato su Whigs e Tories. Questa divisione rappresentò nel corso del XVIII e XIX secolo la netta distinzione tra un vecchio ordine ancora precapitalista (il partito Tory) e il nuovo mondo liberista e "radical-borghese" (i Whigs). Il sistema economico loro contemporaneo era quello del trionfo Whig, del capitalismo del laissez faire che aveva assorbito - da un punto di vista ideale - anche l'antico partito conservatore. Nella transizione socialdemocratica, il partito liberale era al tempo stesso baluardo della conservazione (del modello capitalista) e del progresso (la democratizzazione, il diritto di voto, le riforme politiche). In questo senso, i socialisti dovevano lavorare con i Whigs per ampliare la democrazia, ma al tempo stesso esserne anche avversari irriducibili avendo come obiettivo il superamento della società borghese. Nell'interessante analisi di Hubert Bland, con l'avvenuta omogeneità della rappresentanza politica rispetto agli interessi economici attraverso la formazione di un partito socialista, la componente più "di sinistra" del partito liberale vi sarebbe confluita, mentre la destra liberale sarebbe entrata nel partito conservatore, oramai intrinsecamente favorevole al capitalismo ${ }^{18}$. In

means the gradual extension of the franchise; and the transfer of rent and interest to the State, not in one lump sum, but by instalments.» sottolineando anche come «A democratic State cannot become a Social-Democratic State unless it has in every centre of population a local governing body as thoroughly democratic in its constitution as the central Parliament.», pp. 182, 188.

${ }^{15}$ SHAW, George Bernard, Transition, cit., p. 180.

${ }^{16}$ Ibidem, pp. 187-201.

${ }^{17}$ BLAND, Hubert, The Outlook, in SHAW, George Bernard, et. al., Fabian Essays in Socialism, cit., pp. 202-220.

${ }^{18}$ Ibidem, pp. 212-220. 
questo modo, l'anima progressista del vecchio liberalismo politico sarebbe stata raccolta dal socialismo, come sarebbe stato auspicato anche da Rosselli in riferimento al compito "storico" del movimento socialista, nella convinzione che in qualche modo esso assorbisse dentro di sé la funzione liberale ${ }^{19}$.

Nel vangelo fabiano del 1889, l'arrivo del socialismo era insomma visto come una spinta intrinseca dello stesso metodo di produzione capitalista, ma - ed è qui che forse si giocava una sostanziale differenza rispetto all'interpretazione marxista "ortodossa" allo stesso tempo essa doveva essere accompagnata da un movimento di riforma democratico, graduale, costituzionale, pacifico e che investisse ogni aspetto della vita sociale degli individui.

Nicola Tranfaglia ha accennato ad una qualche influenza del fabianesimo nel pensiero di Rosselli ${ }^{20}$. Quest'influenza appare rilevante anzitutto nella concezione del socialismo come una rivoluzione "morale", un vero e proprio "programma di vita da attuare» ${ }^{21}$, piuttosto che come un programma economico ricavato da un'ideologia. Anche la visione gradualistica e la "funzione" di democrazia sostanziale del socialismo possono essere punti di convergenza tra fabianesimo e socialismo liberale, per cui «il socialismo non è che lo sviluppo logico, sino alle sue estreme conseguenze, del principio di libertà» ${ }^{22}$.

Tuttavia, se il socialismo di Rosselli era ancora fondamentalmente gradualista, egli negava fortemente qualsiasi necessità storica del socialismo come contraddizione dei mezzi di produzione (nella sua variante marxista) o come necessità morale e sociale (come per i fabiani), poiché egli anzi affermava che «il regime socialista sarà, ma potrebbe anche non essere. Sarà se noi lo vorremmo, se le masse vorranno che sia, attraverso un consapevole sforzo creatore» 23 .

Rosselli è vissuto in un'altra epoca e in un altro luogo rispetto alla Gran Bretagna di fine XIX secolo; rispetto agli anni di sereno ottimismo dei fabiani, quelli di crisi tra le

19 Tra i numerosi scritti in cui Rosselli esprime questa sua convinzione sul liberalismo come "metodo" si veda, se non altro per la loro "importanza", ROSSELLI, Carlo, «Liberalismo socialista», in La Rivoluzione liberale, 15 luglio 1924, in ID., Socialismo liberale e altri scritti, ROSSELLI, John (a cura di), Torino, Einaudi, 1973, pp. 107-128; si veda anche, nel contesto storico dell'esilio francese, ID., «Liberalismo rivoluzionario», in Quaderni di Giustizia e Libertà, 1, gennaio 1932, Torino, Bottega d'Erasmo, 1959, pp. 25-29.

${ }^{20}$ TRANFAGLIA, Nicola, Carlo Rosselli dall'interventismo a Giustizia e Libertà, Bari, Laterza, 1968, p. 159.

${ }^{21}$ ROSSELLI, Carlo, Socialismo Liberale, in ID., Socialismo liberale e altri scritti, cit., p. 432,

${ }^{22}$ Ibidem, p. 436. Su questi aspetti si vedano i fondamentali capitoli V, VI e VII di Socialismo Liberale, pp. 419-468. Lo stesso Cole si sarebbe più tardi definito un socialista "liberale", e la Società Fabiana come un gruppo di socialisti "liberali", nel senso di non ortodossi e dogmatici. COLE, G. D. Howard, Il Socialismo fabiano, Milano, La Fiaccola, 1943 [edizione clandestina], p. 6.

${ }^{23}$ ROSSELLI, Carlo, Socialismo Liberale, in ID., Socialismo liberale e altri scritti, cit., p. 455. 
due guerre non permettevano più di ritenere il socialismo la felice conclusione della trasformazione pacifica e inevitabile della società, tanto più per una generazione come quella di Rosselli, diventata adulta con la Grande Guerra24.

Tuttavia ancora in Socialismo Liberale era prospettato uno sbocco "socialista" della crisi del capitalismo, rifacendosi (criticamente) ai più avanzati settori "revisionisti" del socialismo europeo, da Bernstein a De Man²5, allo stesso sindacalismo di Howard Cole, citato nell'opera ${ }^{26}$.

\section{Tra democrazia industriale e sindacalismo gildista}

Gli intellettuali laburisti fabiani esercitarono una certa influenza su Rosselli proprio nell'elaborazione della sua "teoria" sindacale ${ }^{27}$. Il primo viaggio fatto dal giovane Rosselli dall'agosto all'ottobre del 1923 lo mise in contatto con i maggiori esponenti della Fabian Society, tra cui proprio i Webb e Howard Cole. Essi rappresentavano anche due diverse prospettive di ideologia sindacale: la democrazia industriale da un lato, il controllo operaio dall'altro ${ }^{28}$.

\footnotetext{
${ }^{24}$ Sulla prima formazione politica di Carlo Rosselli, si veda TRANFAGLIA, Nicola, op. cit., pp. 11-71; sul militante politico come "uomo d'azione", si veda ROSSELLI, Carlo, «Volontarismo», in Il Quarto Stato, 12 giugno 1926, ora in ID., Socialismo liberale e altri scritti, cit., pp. 145-147.

${ }^{25}$ BERNSTEIN, Eduard, I presupposti del socialismo e $i$ compiti della socialdemocrazia, RomaBari, Laterza, 1968 [ed. originale: Die Voraussetzungen des Sozialismus und die Aufgaben der Sozialdemokratie, Stuttgart, J.H.W. Dietz, 1899]; DE MAN, Henri, Il superamento del marxismo, 2 voll., Bari, Laterza 1929, [ed. originale: Au-delà du marxisme, Paris, Alcan, 1929]; sull'influenza del pensiero revisionista su Carlo Rosselli, in particolare di De Man e dei neosocialisti di Déat si veda DEGL'INNOCENTI, Maurizio, Socialismo liberale e socialismo europeo, in ID. (a cura di), Carlo Rosselli e il socialismo liberale, Manduria, Manduria-RomaBari, 1999, pp. 65-108; per uno sguardo complessivo sul socialismo europeo tra le due guerre, si veda PANACCIONE, Andrea, Socialisti europei. Tra guerre, fascismo e altri catastrofi (19121946), Milano, Franco Angeli, 2000.

${ }^{26}$ ROSSELLI, Carlo, Socialismo Liberale, in ID., Socialismo liberale e altri scritti, cit. Sul revisionismo socialista cfr. pp. 370-418. Sul sindacalismo di Cole cfr. pp. 444-445.

${ }^{27} \mathrm{Su}$ questo aspetto del pensiero di Rosselli, si veda DEGL'INNOCENTI, Maurizio, Carlo Rosselli e il movimento sindacale: dalla tesi di laurea a "Socialismo Liberale", in Giustizia e Libertà nella lotta antifascista e nella storia d'Italia. Attualità dei Fratelli Rosselli a quaranta anni dal loro sacrificio (Atti del Convegno Internazionale organizzato a Firenze 1o-12 giugno 1972), Firenze, La Nuova Italia, 1978, pp. 49-68.

${ }_{28}$ Sull'influenza fondamentale dei Webb, e soprattutto di Howard Cole, si veda anche CALABRÒ, Carmelo, Liberalismo, democrazia, socialismo. Lïtinerario di Carlo Rosselli, Firenze, Firenze University Press, 2009, pp. 58-71. Per un confronto tra gildismo e democrazia industriale, si veda MARUCCO, Dora, Fabianesimo, ghilidsmo, forme di democrazia industriale, Milano, Franco Angeli, 1986, cfr. pp. 9-30.
} 
I Webb studiarono la storia delle Trade Unions per fondare la loro teoria della democrazia industriale, incardinata sul "dogma” dell'efficienza produttiva e sulla funzione democratica svolta dal sindacalismo britannico ${ }^{29}$.

Nella loro analisi, la capacità produttiva poteva essere incrementata dall'attivazione di una legislazione sociale che garantisse almeno il salario minimo e alcune normative sulle condizioni del posto di lavoro a livello nazionale, spostando il "cuore" della lotta sindacale dai salari alla qualità della produzione e alla concorrenza tra le industrie. Un'istituzione economica centrale doveva essere la contrattazione collettiva, in modo da garantire la maggior efficienza, e quindi la maggior competitività, delle imprese in cui vi fosse stato un vincolo normativo e legale tra l'imprenditore e gli operai. La lotta di classe poteva così essere superata da un nuovo sistema industriale pubblico/privato e "corporativo", attentamente diretto dallo Stato e stimolato dall' emergere di industrie concorrenziali il cui indirizzo in merito alle scelte produttive sarebbe stato determinato dalle associazioni di consumatori e, soprattutto, dalle municipalizzate e dalle imprese di proprietà statale.

La crescita del tradunionismo avrebbe inoltre prodotto notevoli conseguenze nella stessa democrazia politica. In Gran Bretagna i sindacati hanno esercitato una funzione di regolazione del conflitto industriale, di mutuo soccorso e di consulenza tecnicoamministrativa. Le Trade Unions erano così in grado di fornire un modello per la stessa composizione dei conflitti sociali ed economici tra classi sociali e tra categorie di produttori all'interno dello Stato, che sarebbe così maturato da una democrazia "politica" ad una democrazia "industriale". Tali conflitti sarebbero stati armonizzati e diretti dallo Stato centrale, primariamente attraverso la legislazione sociale che sarebbe così diventata il fulcro della nuova organizzazione economica e sociale ${ }^{30}$.

Il socialismo gildista di Cole si concentrava invece maggiormente sulla questione della libertà e dell'autogestione operaia, caratterizzandosi per il suo spirito antiautoritario e decentratore. La teoria sociale gildista intendeva ricostruire un nuovo ordine politico ed economico dalla gestione industriale, e non si basava sull'ideologia, ma sulla questione sociale per come essa «presents itself today» ${ }^{1}$. Era una teoria democratica e federalista, perché si proponeva di estendere il principio democratico dalle istituzioni politiche a quelle socio-economiche, e attraverso l'autogoverno dal

\footnotetext{
${ }^{29}$ BERTA, Giuseppe, I Webb e lo studio del tradeunionismo, in WEBB, Sidney, WEBB Beatrice, La democrazia industriale. Antologia degli scritti, Roma, Ediesse, 1994, pp. 9-60, cfr. in particolare pp. 40-60.

${ }^{30}$ WEBB, Sidney, WEBB, Beatrice, La democrazia industriale, Torino, UTET, 1912, pp. 565-773.

${ }^{31}$ COLE, G. D. Howard, Guild Socialism Restated, Piscataway, Transaction Publishers, 1980, p. 11.
} 
mondo dell'industria a quello delle comunità. Da un punto di vista "politico", la soluzione richiamata era un progetto di tipo sindacalistico-corporativo basato sulle organizzazioni di produttori che si richiamavano vagamente alle antiche "gilde" medioevali o artigiane. Tale richiamo era però, nella teoria di Cole, puramente nominale poiché esse avrebbero anche ricoperto il ruolo di rappresentanza delle categorie dei produttori nello "Stato gildista". La soluzione di Cole era fortemente critica verso le tendenze marxiste o socialiste riformiste che operavano all'interno della logica statale, poichè queste si limitavano al controllo del potere statale per realizzare il socialismo ${ }^{32}$.

In un articolo pubblicato su La Rivoluzione liberale ${ }^{33}$, Rosselli ha analizzato la problematicità di queste ipotesi alternative per il sindacalismo britannico.

Secondo la sua interpretazione, il postulato da cui partivano i Webb era che una cooperativa di produttori/consumatori da un punto di vista produttivo non fosse inferiore alle imprese privat - mentre era certamente moralmente superiore costituendo un organo democratico in cui i produttori sarebbero stati anche i consumatori di ciò che producevano, senza fini di "profitto", come tradizionalmente inteso34. Ben lungi dall'essere una soluzione utopistica, le cooperative erano destinate ad estendere le loro attività dall'industria e dall'agricoltura, al credito ed ai servizi. Nella disamina fatta da Rosselli, le cooperative erano viste come le cellule di una società costituita da molteplici organizzazioni imprese collettive, gerarchiche al loro interno e concorrenziali tra loro. I due maggiori limiti, secondo Rosselli, erano proprio la logica "capitalistica" basata sul consumo e il mantenimento dello sfruttamento del lavoro salariato: «[...] nella cooperativa di produzione si lavora per il profitto, per il massimo profitto; è un egoismo a basi più larghe dell'attuale che si organizza», e inoltre, nonostante la "socializzazione" delle imprese in organi cooperativi, «[...] il salariato però non scomparirebbe in un regime a cooperazione universalizzata» 35 .

Diverso era invece l'approccio di Cole, che guardava alla questione industriale come ad un problema «di coscienza, di dignità, di libertà» 36 . Gli aspetti più convincenti del gildismo, secondo Rosselli, erano il superamento di una logica organizzativa basata sul consumo e il conferimento della proprietà dei mezzi di produzione ai produttori stessi. Disinteressandosi inoltre al problema dello Stato, limitato al semplice aspetto politico,

\footnotetext{
${ }^{32}$ Ibidem, pp. 9-62 e 174-207.

${ }^{33}$ ROSSELLI, Carlo, «Il movimento operaio», in La Rivoluzione liberale, 25 marzo 1924, ora in ID., Socialismo liberale e altri scritti, cit., pp. 65-76.

${ }^{34}$ Ibidem, pp. 67-70.

${ }^{35}$ Ibidem, p. 69 e 68.

${ }^{36}$ Ibidem, p. 71.
} 
il gildismo poteva imprimere anche ulteriore forza ad un movimento sindacale nell'ambito di una più ampia e graduale estensione delle sue funzioni per la compartecipazione all'impresa, fino alla direzione indipendente. L'organizzazione che ne conseguiva era infine anch'essa cooperativa, ma su base federale, nazionale e per categoria di produzione (a differenza delle cooperative "municipali" dei Webb), completamente autonoma al proprio interno (nell'amministrazione, nei rapporti tra aderenti), ma vincolata ad organi misti tra gilde e amministrazioni pubbliche (che sarebbero rimaste in essere)37. Infine altro aspetto da non sottovalutare era la perfettibilità del sistema gildista, il quale, con un'espressione tipica di Rosselli, si sarebbe potuto migliorare attraverso «l'esperienza, liberamente attuata,» che «coi suoi risultati magari dapprima dolorosi e negativi, potrà indicarci la via nuova degli anni a venire» 38 .

Gli esiti di questo primo viaggio, motivato proprio dalla volontà di approfondire le sue conoscenze scientifiche e ideologiche della teoria economica e sindacale, avrebbero rinsaldato gli entusiasmi di Rosselli nei confronti del movimento laburista, nonostante la sua consapevolezza delle difficoltà del movimento in quel momento storico. Secondo Tranfaglia, egli guardava «al laburismo e all'ideologia a cui in una certa misura il movimento s'ispira, come alla soluzione socialista dell'avvenire»39, anche in virtù dell'azione concreta del partito laburista.

\section{Il Labour Party e le Trade Unions come modelli per il movimento operaio}

Il viaggio dell'anno successivo si svolse nel momento in cui la situazione italiana precipitava drammaticamente in seguito al delitto Matteotti e alla secessione dell'Aventino. Il suo interesse era quindi maggiormente incentrato sulla strategia politica laburista verso i ceti medi durante le elezioni politiche del 1924; ceti medi che in Italia invece stavano andando ad ingrossare le fila del fascismo. Rosselli fu testimone diretto del confronto elettorale tra laburisti e conservatori, che delineava proprio in quegli anni il futuro schiacciamento dei liberali nel sistema bipolare britannico. Il suo ruolo fu di "corrispondente" dell'evoluzione della situazione politica inglese, in stretta relazione con quella italiana.

\footnotetext{
${ }^{37}$ Ibidem, pp. 70-74.

${ }^{38}$ Ibidem, p. 76.

39 TRANFAGLIA, Nicola, op. cit., p. 126.
} 
Rosselli partiva dal presupposto che il partito laburista inglese fosse un partito completamente differente rispetto ai suoi corrispettivi continentali, poiché era nato «spontaneamente, come fatto elementare, frutto diretto della rivoluzione industriale. Mentre sul continente è l'idea, l'ideologia marxista che attraverso i Partiti crea l'organizzazione economica [...]»40. 亡̀ forse proprio questo aspetto che maggiormente colpì Rosselli, ossia il rifiuto di «ogni ideologia aprioristica»41. La creazione del partito in Gran Bretagna avvenne, a suo giudizio, quasi per costrizione, quando gli interessi economici e corporativi della classe operaia, organizzatasi "dal basso", trovarono una rappresentanza politica per poter essere meglio difesi. Lo stesso partito laburista ricalcava l'impostazione "federalista" del movimento operaio inglese, che passava dalle Trade Unions comuniste a quelle che semplicemente tutelavano i propri interessi di categoria. Questa natura "composita”, si rifletteva in una rappresentanza politica che ha sempre inteso tutelare «tutto il mondo del lavoro [...] non in nome e nell'interesse di una sola classe, ma in nome e nell'interesse di tutto il paese col metodo democraticoliberale», costituendo così «un partito costituzionale socialisteggiante, che va dalla sinistra liberale ai comunisti» 42 .

Una così ampia base di interessi è stata tenuta unita da una prospettiva socialista che presentava alcuni tratti peculiari rispetto al socialismo marxista "continentale", che rifiutava la lotta di classe da un punto di vista dottrinale - pur praticandola de facto indirizzandosi verso un programma socialistico riformista, municipale ma pur sempre collettivista 43 .

La pratica liberaldemocratica del partito laburista fu evidente durante le elezioni del 1924: uscente era proprio la prima storica amministrazione laburista, presieduta da Ramsay MacDonald. Il governo era sopravvissuto soltanto pochi mesi grazie al sostegno liberale 44 , e la sua crisi portò a nuove elezioni, seguite da Rosselli in una ricostruzione giornalistica certamente parziale, ma interessante per ricostruire a quale tipo di partito Rosselli pensasse come modello per l'Italia45.

\footnotetext{
${ }^{40}$ ROSSELLI, Carlo, «Il Partito del Lavoro in Inghilterra», in Libertà, anno I, 15 febbraio 1924, in MASTELLONE, Salvo, op. cit., p. 142.

${ }^{41}$ Ibidem.

${ }^{42}$ Ibidem, p. 148.

${ }^{43}$ Ibidem, pp. 146-148.

${ }^{44}$ CLARKE, Peter, Speranza e gloria. L'Inghilterra nel XX secolo, Bologna, Il Mulino, 2000, pp. 160-163.

${ }^{45} \mathrm{Si}$ vedano gli articoli di Carlo Rosselli per «La Giustizia» sulle elezioni inglesi. ROSSELLI, Carlo, «Le ragioni degli accordi tra liberali e conservatori», in La Giustizia, 17 ottobre 1924; «Laburisti e liberali faccia a faccia», in La Giustizia, 21 ottobre 1924; "Un messaggio di MacDonald per "La Giustizia"», in La Giustizia, 23 ottobre 1924; "I laburisti torneranno alla Camera più forti di prima», in La Giustizia, 24 ottobre 1924; "I fattori della vittoria
} 
Dalle sue interviste, si desume la consapevolezza da parte laburista di trovarsi ad un tornante fondamentale della propria storia politica. Da un lato, si verificò la convergenza di alcuni liberali e dei conservatori su posizioni molto simili tra loro; dall'altro, i laburisti dimostrarono di essere in grado di governare, a livello locale e centrale, spuntando l'arma propagandistica del partito "antisistema", dimostrandosi, al contrario, in grado di rappresentare le masse lavoratrici, escluse invece proprio dagli interessi minoritari ma meglio organizzati del blocco liberale-conservatore ${ }^{46}$. Oltretutto, dando implicitamente per scontata una vittoria conservatrice 47 , la vera posta in gioco sarebbe stata l'avanzata laburista e la scomparsa liberale. Secondo le previsioni di Rosselli, questa lotta si sarebbe risolta a vantaggio del Labour Party, cioè «di quelle forze che esso stesso [il partito liberale] aveva sprigionato colla sua azione riformatrice» ${ }^{4}$. Il percorso storico era inevitabile perché, secondo la visione "tipica” del liberalismo proposta da Rosselli: «la specifica funzione progressista che il Partito Liberale aveva compiuto per tutto il secolo XIX nel campo politico, va passando inevitabilmente al Partito Labourista che si propone di assolvere una analoga e grandiosa funzione specie sul terreno economico» 49 .

Questa tendenza di lungo periodo, già prevista dal fabiano Bland alla fine del XIX secolo, è stata comunque incentivata da due fattori: la contraddizione tra un partito che si professava progressista, ma che in campo economico - nella sua ala "di destra" - era conservatore e borghese e l'esperienza di governo di MacDonald. Quest'ultimo ha dimostrato come il Labour fosse in grado di governare, senza per questo tradire il programma di riforme socialistiche, come ad esempio sulla legislazione del lavoro ${ }^{50}$.

Fu proprio "l'eliminazione" del partito liberale il cosciente obiettivo laburista; in un'intervista all'esponente della sinistra del partito, Lansbury, questi affermava senza mezzi termini come, con le elezioni del 1924, l'asino "a due teste", cioè il capitalismo, avesse perso definitivamente i Whigs. La storia del radicalismo inglese, e vasta parte dell'elettorato liberale - nelle previsioni del parlamentare laburista - in futuro sarebbero state espresse dal Labour, che avrebbe rappresentato «le coscienze progressiste», le quali «si uniscono allo scopo di realizzare quei mutamenti economici

conservatrice», in La Giustizia, 1 novembre 1924, tutti ora in MASTELLONE, Salvo, op. cit., pp. 159-173.

${ }^{46}$ ROSSELLI, Carlo, «Le ragioni degli accordi tra liberali e conservatori», cit., cfr. pp. 160-161.

${ }^{47}$ Secondo i laburisti ciò sarebbe stato dovuto anche ad una campagna giocata sui presunti legami dei laburisti con l'Unione Sovietica e all'espressione di un voto in larg parte conservatore da parte delle donne. ID., «I fattori della vittoria conservatrice», cit.

${ }^{48}$ ID., «Laburisti e liberali faccia a faccia», cit., p. 163.

${ }^{49}$ Ibidem.

${ }^{50}$ Ibidem, pp. 164-166. 
senza i quali la libertà politica si risolve in una mera finzione» ${ }^{1}$, e allo stesso tempo anche «Tutti i lavoratori, del braccio o del cervello, [...]»52. Quest'ultimo aspetto è molto importante per la rilevanza che l'impostazione non-classista, ma al contrario volta alla massima inclusività possibile, ha avuto nel pensiero politico di Rosselli e che gli valse in tempi diversi le critiche non solo dei comunisti, ma anche dei socialisti riformisti italiani53.

È interessante così, come rileva sempre Tranfaglia, sottolineare come la "lezione" che Rosselli trasse dall'azione politica del partito laburista fosse per l'appunto la soluzione sostanzialmente non classista e centrista, che, ancora in quegli anni, era l'orizzonte politico entro cui si muoveva la sua analisi54.

La situazione politica inglese sarebbe rimasta comunque sempre un termine di riferimento per Rosselli. Anche nel 1926, con il proseguire della crisi del socialismo accompagnata dal rafforzamento del fascismo, dalle pagine del Quarto Stato Rosselli criticava il dottrinarismo del partito socialista di fronte al fascismo55. Tale limite si rivelava, secondo lui, nell'incapacità di organizzare politicamente l'opposizione al regime e nel dogmatismo marxista-riformista, che risultava inadeguato nel comprendere la natura stessa del fascismo e contemporaneamente "svalutava" i valori democratici, senza per giunta riuscire a dimostrarsi una forza coerente, in grado di assumere il potere, «alfa e omega del movimento proletario» 56 .

Di contro, lo sciopero generale britannico del 1926 - passato alla storia proprio come "lo sciopero generale" 57 - avrebbe permesso di apprendere due lezioni. La prima

\footnotetext{
${ }^{51}$ ID., «I laburisti torneranno alla Camera più forti di prima», cit., p. 169.

${ }^{52}$ Ibidem, p. 170.

${ }^{53} \mathrm{Si}$ veda la polemica con il socialista Saragat e con il comunista Grieco in merito al programma agrario di G.L. nel 1932. Si veda [ROSSELLI, Carlo], «Il critico di sé stesso», in Quaderni di Giustizia e Libertà, 2, marzo 1932, pp.16-17, ID., "Discussioni sul programma agrario», in Quaderni di Giustizia e Libertà, 4, settembre 1932, pp. 76-86.

54 Tranfaglia dedica alcune pagine ai due viaggi di Carlo Rosselli in Gran Bretagna, sottolineando per l'appunto il carattere più "accademico" del primo e giornalistico del secondo. TRANFAGLIA, Nicola, op. cit., pp. 159-164 e pp. 188-190.

${ }^{55} \mathrm{Su}$ questo tema si veda BAGNOLI, Paolo, La battaglia socialista de "Il Quarto Stato", in Giustizia e Libertà nella lotta antifascista e nella storia d'Italia. Attualità dei Fratelli Rosselli a quaranta anni dal loro sacrificio (Atti del Convegno Internazionale organizzato a Firenze 1012 giugno 1972), cit., pp. 113-146; MERLI, Stefano, "Il "Quarto Stato" di Rosselli e Nenni e la polemica sul rinnovamento socialista nel 1926», in Rivista storica del socialismo, 11, 3/1960, pp. 19-28.

${ }^{56}$ ROSSELLI, Carlo, «Autocritica, non demolizione», in Il Quarto Stato, 1 maggio 1926, ora in ID., Socialismo liberale ed altri scritti, cit., pp. 133-144, citazione a p. 137; sulla stessa lunghezza d'onda, si veda anche ID., "Autocritica», in Il Quarto Stato, 3 aprile 1926, ora in ID., Socialismo liberale ed altri scritti, cit., pp. 129-132.

${ }_{57}$ Questo sciopero fu causato dai tentativi dei proprietari delle miniere di ridurre i salari e aumentare le ore lavorative. Il 3 maggio il TUC decise di proclamare uno sciopero di solidarietà con i minatori: nel settore dei trasporti (ferrovieri in primis), dell'energia, della chimica, della siderurgia, dell'edilizia, della meccanica, dei cantieri marittimi, dell'edilizia e della tipografia.
} 
era che il lungo sciopero generale attuato dai lavoratori inglesi diventava non più una rivendicazione economica, bensì «un fatto squisitamente rivoluzionario» 58 . Era cioè uno strumento di lotta intrinsecamente politico; Rosselli citava nell'articolo Bertrand Russell, secondo cui lo sciopero poteva avere «un posto ben definito nella strategia socialista. [...] nella resistenza al fascismo, dopo una vittoria conseguita, attraverso mezzi democratici, da un governo socialista»59. Nell'ambito della ricerca di un rinnovamento socialista ne «Il Quarto Stato», l'implicito suggerimento di Rosselli era quello di sottolineare l'importanza che l'uso dello sciopero generale - dimostrare cioè "da che parte stavano" i lavoratori italiani - poteva ancora avere come mezzo per riorganizzare le masse da parte dei socialisti nella lotta contro il fascismo.

L’insegnamento che si può trarre dallo sciopero inglese è tuttavia di carattere più generale. Il risultato dello sciopero generale poteva difatti fornire un riscontro all'ipotesi per la quale - in condizioni di legalità democratica - «la democrazia borghese permette il graduale e possente avanzarsi delle forze di lavoro» 60 .

Effettivamente, le elezioni del 1924 rappresentarono una svolta per il partito laburista, che avrebbe allargato i suoi elettori dai soli iscritti ai sindacati al ceto medio; questa ultima classe sociale avrebbe composto in misura sempre più rilevante il gruppo dirigente e parlamentare. Le stesse elezioni segnarono anche l'inizio del declino Whig come seconda forza politica in Gran Bretagna.

Questo fenomeno, ricostruito egregiamente da Rosselli come un "riposizionamento" ed un chiarimento nello schieramento tra forze capitaliste ed anticapitaliste, così come venne inteso dagli stessi laburisti all'epoca, fu il prodotto del sistema elettorale uninominale. Il partito liberale aveva storicamente avuto una rappresentanza sostanzialmente omogenea, distribuita su tutto il Paese, laddove invece laburisti e conservatori avevano piuttosto dei "feudi" elettorali nei collegi operai e borghesi. La

Per 9 giorni lo sciopero coinvolse un milione e mezzo di lavoratori, mentre i minatori avrebbero continuato lo sciopero per altri 6 mesi. Si veda CLARKE, Peter, op. cit., pp. 178-181.; si veda anche PELLING, Henry, Storia del sindacalismo inglese, Milano, Palazzi Editore, 1972, pp. 208-216.

${ }^{8}$ ROSSELLI, Carlo, «L'insegnamento dello sciopero generale inglese», in Il Quarto Stato, 17 luglio 1929, in MASTELLONE, Salvo, op. cit., pp. 177-180, citazione a p. 178.

59 Ibidem, p. 180. Sull'effettiva azione in politica estera del partito laburista in seno all'Internazionale operaia socialista e alla Società delle Nazioni tra anni Venti e anni Trenta, e fino allo scoppio della II Guerra Mondiale, si veda RAPONE, Leonardo, «Il socialismo internazionale, l'ascesa del nazismo e la politica del disarmo", in Studi storici: rivista trimestrale dell'Istituto Gramsci, 37, 4/1996, pp. 1155-1199; TOBIA, Bruno, «Politica estera socialista e problema del fascismo durante l'epoca di Locarno» e MANCINI, Mario, «La politica internazionale del laburismo inglese nella seconda metà degli anni trenta (marzo 1936settembre 1939)», entrambi in Storia contemporanea: rivista bimestrale di studi storici, 11, 45/1980, rispettivamente pp. 709-746 e pp. 747-857.

${ }^{6}$ ROSSELLI, Carlo, «Battaglia storica», in Il Quarto Stato, 8 maggio 1926, in ID., Socialismo liberale ed altri scritti, cit., pp. 77-79. 
sorte del partito liberale fu così notevolmente segnata: se da una parte era il secondo partito nazionale con il 30\% dei consensi, al contempo i singoli candidati liberali nella gran parte dei collegi - persero contro gli altri candidati, fossero essi conservatori o laburisti. Nel 1924, invece, l'esperienza governativa moderata di MacDonald con il sostegno Whig, portò alla sparizione del partito liberale come forza politica presente sul territorio nazionale, poiché quest'ultimo perse il 12\% dei voti e ben 118 parlamentari, il tutto a vantaggio dei conservatori ${ }^{61}$.

\section{Conclusioni}

Howard Cole ha scritto che «il tipico pensatore socialista britannico considera il movimento socialista come l'erede di una tradizione democratica e liberale che è profondamente radicata nel popolo britannico» ${ }^{62}$. Un'esperienza intellettuale così "lontana" e per certi aspetti così diversa rispetto alla realtà del socialismo italiano in cui Rosselli viveva, non gli ha impedito di ricavare anche dall'esperienza laburista britannica una concezione del socialismo come dell'erede storico del liberalismo democratico ed anzi, come l'ampliamento dalle libertà politiche e civili, da affiancare a quelle sociali. Questa convinzione fu esposta, anche in termini di rappresentanza politica, nell'idea che Lansbury espresse a Rosselli di un partito che rappresentasse il mondo del lavoro in termini non classisti, ma comunque contrapposti al profitto del grande capitalismo. Quest’idea sarebbe poi entrata nel patrimonio politico giellista come un suo tratto peculiare.

Carlo Rosselli ha successivamente avuto un percorso politico e biografico che lo portò sempre più a sinistra ${ }^{63}$. Tuttavia, alcune tematiche di fondo del suo pensiero, come l'autogestione operaia, la funzione liberale del socialismo, il pragmatismo, il volontarismo e la democrazia sostanziale, contrapposta a quella formale erano già presenti sin dalla sua prima formazione politica,ed emersero anche dal confronto con l'esperienza politica inglese, per certi aspetti tra le più avanzate d'Europa.

\footnotetext{
${ }^{61}$ Si veda CLARKE, Peter, op. cit., pp. 152-164.

${ }^{62}$ COLE, G. D. Howard, «Gli elementi non marxisti nel socialismo britannico», in Il Ponte, 8, 56/1952, pp. 531-539, citazione p. 537.

${ }^{63}$ Una sintesi di tale evoluzione in TRANFAGLIA, Nicola, Sul socialismo liberale di Carlo Rosselli, in I dilemmi del liberalsocialismo, cit., pp. 85-104, in cui l'autore ipotizza una sorta di "comunismo liberale" nel periodo finale della vita di Rosselli, nell'indicazione del proletariato come forza fondamentale della rivoluzione antifascista e nella ricerca di un'alleanza stabile con il partito comunista.
} 


\section{* L'autore}

Michele Mioni ha conseguito la laurea in "Storia dal Medioevo all'Età contemporanea" presso l'Università Ca'Foscari di Venezia, con una tesi dal titolo L'evoluzione delle politiche di welfare: il caso britannico dal governo Attlee al New Labour. I suoi principali interessi di ricerca vertono attorno alla storia economica e sociale del XX secolo e alla storia dei movimenti politici contemporanei. Su Carlo Rosselli e il movimento giellista in Francia, ha pubblicato «I Quaderni di Giustizia e Libertà (1932-1935): un laboratorio di politica», in Quaderni del Circolo Rosselli, 3/2011, pp. 195-236.

URL: < http://studistorici.com/progett/autori/\#Mioni >

\section{Per citare questo articolo:}

MIONI, Michele, «L'esperienza intellettuale e politica del laburismo nel pensiero di Carlo Rosselli», Diacronie. Studi di Storia Contemporanea: Sulle tracce delle idee, 29/12/2012,

URL:< http://www.studistorici.com/2012/12/29/mioni_numero_12/ >

Diacronie Studi di Storia Contemporanea 3 ww.diacronie.it

Risorsa digitale indipendente a carattere storiografico. Uscita trimestrale.

redazione.diacronie@hotmail.it

Comitato di redazione: Marco Abram - Jacopo Bassi - Luca Bufarale - Gianluca Canè - Alessandro Cattunar - Alice De Rensis - Barbara Galimberti - Deborah Paci - Fausto Pietrancosta - Matteo Tomasoni - Luca Zuccolo

Diritti: gli articoli di Diacronie. Studi di Storia Contemporanea sono pubblicati sotto licenza Creative Commons 2.5.

Possono essere riprodotti a patto di non modificarne i contenuti e di non usarli per fini commerciali. La citazione di

estratti è comunque sempre autorizzata, nei limiti previsti dalla legge. 